\title{
Comprehensive analysis of long non-coding RNA and mRNA expression profiles in rheumatoid arthritis
}

\author{
QING LUO ${ }^{1 *}$, CHUXIN XU², XUE LI ${ }^{2}$, LULU ZENG $^{2}$, JIANQING YE $^{2}$, \\ YANG GUO $^{2}$, ZIKUN HUANG ${ }^{1}$ and JUNMING LI ${ }^{1}$ \\ ${ }^{1}$ Department of Clinical Laboratory, The First Affiliated Hospital of Nanchang University; \\ ${ }^{2}$ Department of Medical College, Nanchang University, Nanchang, Jiangxi 330006, P.R. China
}

Received November 3, 2016; Accepted August 10, 2017

DOI: $10.3892 / \mathrm{etm} .2017 .5284$

\begin{abstract}
Abnormal expression of long non-coding RNA (lncRNA) has been demonstrated to be involved in a variety of human diseases. However, the role of lncRNA remains largely unknown in rheumatoid arthritis (RA). The present study aimed to investigate whether IncRNA are differentially expressed in RA. Differentially expressed lncRNA and mRNA in peripheral blood mononuclear cells from individuals with RA and healthy controls were detected using a human lncRNA microarray containing 30,586 lncRNA and 26,109 coding transcripts. Several candidate lncRNA and mRNA in 24 paired samples were verified by reverse transcription-quantitative polymerase chain reaction analysis. Bioinformatics analyses (Gene Ontology and Kyoto Encyclopedia of Genes and Genomes) were used to evaluate signaling pathways and biological functions influenced by the differentially expressed mRNA. A total of 5,045 lncRNA (upregulated, 2,410; downregulated, 2,635) and 3,289 mRNA (upregulated, 1,403; downregulated, 1,886) were differentially expressed in patients with RA (fold-change $>2 ; \mathrm{P}<0.05$ ). The majority of abnormal lncRNA were from intergenic spacer regions (42\%), natural antisense (19\%) and intronic antisense $(15 \%)$ to protein-coding loci. lncR NA target prediction indicated the presence of 135 potential lncRNA-mRNA target pairs for the 85 aberrant lncRNA and 109 aberrant mRNA. Significantly enriched $(\mathrm{P}<0.05)$ signaling pathways based on deregulated mRNA were mostly implicated in bile secretion, $\mathrm{T}$ cell receptor signaling pathway and systemic lupus erythematosus.
\end{abstract}

Correspondence to: Dr Junming Li or Dr Zikun Huang, Department of Clinical Laboratory, The First Affiliated Hospital of Nanchang University, 17 Yongwai Zheng Jie, Nanchang, Jiangxi 330006, P.R. China

E-mail: 1isir361@163.com

E-mail: 491353062@qq.com

*Contributed equally

Key words: rheumatoid arthritis, long non-coding RNA, mRNA, peripheral blood mononuclear cells
In summary, to the best of our knowledge, the present study executed global expression profiling of lncRNA and mRNA involved in RA for the first time. These results may provide important insights regarding lncRNA in RA pathogenesis and provide potential therapeutic targets.

\section{Introduction}

Rheumatoid arthritis (RA) is a systemic autoimmune disease that predominantly affects multiple peripheral joints and results in irreversible joint damage (1). Accumulating evidence suggests that the participation of inflammation-associated cells, as well as their production of proinflammatory mediators, including tumor necrosis factor (TNF)- $\alpha$ and interleukin-1 $\beta$, serve a key role in the pathogenesis of RA (2). Although major strides in understanding the disease have been made, the specific mechanistic details underlying the pathogenesis of RA have not been fully clarified (2).

In the human genome, $>70 \%$ is availably transcribed; however, only $1-2 \%$ encodes proteins $(3,4)$. The majority of the remainder of the genome generates non-coding RNA (ncRNA $(5,6)$. According to their length, ncRNA may be divided into long ncRNA (lncRNA), which are arbitrarily defined as transcripts longer than $200 \mathrm{nt}$, or short ncRNA, including microRNA (miRNA) and short interfering RNA (7). It is well documented that miRNA-155, -146 and -21 are aberrantly expressed in RA (8-10), suggesting that miRNA serve critical roles in the regulation of RA pathogenesis. An increasing number of studies have suggested that lncRNA have a critical role in fundamental biological processes, including genomic imprinting, chromosome modification, immune response, inflammatory conditions, tumorigenesis, cellular development and metabolism, through comprehensive mechanisms $(11,12)$. Emerging evidence has also indicated that the expression of lncRNA is dysregulated in a range of disorders, including cancer, intervertebral disc degeneration and Alzheimer's disease (13-15). Additionally, the deregulation of various lncRNA has been implicated in RA, including H19, Hotair, large intergenic non-coding (linc)RNA-p21, C5T11ncRNA, LOC100652951 and LOC100506036 (16-20). Despite these notable developments, the alteration in lncRNA and mRNA expression levels induced by RA and the roles of these transcripts in modulating RA pathogenesis remain unclear. 
The present study determined the expression patterns of lncRNA and mRNA in RA samples and compared them with healthy samples. Additionally, three lncRNA and three mRNA were confirmed using reverse transcription-quantitative polymerase chain reaction (RT-qPCR). lncRNA classification, subgroup analysis and genomic location analysis were performed to further analyze these differentially expressed lncRNA. Gene Ontology (GO) and Kyoto Encyclopedia of Genes and Genome (KEGG) analysis were used to predict the function and signaling pathways affected by the differentially expressed mRNA, which were target genes of aberrantly expressed lncRNA. The present results suggested that IncRNA expression patterns may provide pivotal insights into the pathogenesis of RA.

\section{Materials and methods}

Patients and control individuals. A total of 34 patients with RA (29 females and 5 males; mean age, 53.7 years) admitted to the Department of Rheumatology and Immunology, The First Affiliated Hospital of Nanchang University (Nanchang, China) between October 2014 and April 2016, were enrolled in the present study. The diagnosis of RA was established by the American College of Rheumatology criteria (21). The baseline characteristics of 10 of these patients that were used in microarray analysis are summarized in Table I. For RA patients, Disease activity was calculated using the disease activity score 28 system (22), anti-cyclic citrullinated peptide (CCP) antibody was detected by Anti-CCP Enzyme Immunoassay Test kit (cat. no. EC170502; Shanghai Kexin Biotech Co., Ltd., Shanghai, China) and values $>25 \mathrm{RU} / \mathrm{ml}$ were considered positive. Erythrocyte sedimentation rate was examined by the Westergren method (23), and values $\leq 15 \mathrm{~mm} / \mathrm{h}$ for males and $\leq 20 \mathrm{~mm} / \mathrm{h}$ for females were considered normal. Serum C-reactive protein (CRP) and rheumatoid factor (RF) were measured by nephelometry. Values $>8 \mathrm{mg} / \mathrm{l}$ for CRP and $>20 \mathrm{IU} / \mathrm{ml}$ were considered positive for RF. A total of 34 healthy controls (29 females and 5 males; mean age, 50.2 years) were selected based on no history of autoimmune disease. Among the patients and controls, 10 patients with RA (Table I) and 10 age-matched and sex-matched normal controls were recruited for isolating peripheral blood mononuclear cells (PBMCs) for microarray analysis. The remaining 24 healthy controls and 24 patients with RA were used to validate the reliability of microarray results by RT-qPCR analysis.

The present study was approved by the Ethics Committee of the First Affiliated Hospital of Nanchang University (approval no. 019) and was conducted in compliance with the Declaration of Helsinki. Informed consent was obtained from all participants, including patients and normal controls, prior to initiation of the study.

PBMC collection and RNA extraction. Blood samples were collected in vacutainer tubes containing EDTA, and PBMCs were isolated by centrifugation against a density. Gradient $\left(1,000 \mathrm{x} \mathrm{g}, 20 \mathrm{~min}, 22^{\circ} \mathrm{C}\right)$. Total RNA was isolated from freshly obtained PBMCs using TRIzol reagent (Invitrogen; Thermo Fisher Scientific, Inc., Waltham, MA, USA) according to the manufacturer's protocol. The integrity of the RNA was assessed by electrophoresis on a $2 \%$ denaturing agarose gel and visualized using ethidium bromide. The concentration and quality of the RNA were assessed by absorbance spectrometry measuring absorbance ratios of A260/A280 and A260/A230 using a NanoDrop ND-1000 spectrophotometer (Thermo Fisher Scientific, Inc.).

Microarray and computational analysis. Arraystar Human LncRNA Microarray V3.0 (8x60 K; Arraystar, Inc., Rockville, MD, USA) is designed for the global profiling of human lncRNA and mRNA, and is updated from the previous Microarray V2.0. The sample preparation and microarray hybridization were performed according to the manufacturer's protocol. Briefly, ribosomal RNA was removed from total RNA and subsequently mRNA was obtained using an mRNA-ONLY ${ }^{\mathrm{TM}}$ Eukaryotic mRNA Isolation kit (Epicentre; Illumina, Inc., San Diego, CA, USA). The random priming method (24) was utilized to amplify each sample according to the manufacturer's protocol (Quick Amp Labeling kit; 5190-0442 Agilent Technologies, Inc., Santa Clara, CA, USA; amplification assay was set at $40^{\circ} \mathrm{C}$ for $2 \mathrm{~h}$, followed by $65^{\circ} \mathrm{C}$ for $15 \mathrm{~min}$ and $0^{\circ} \mathrm{C}$ for $5 \mathrm{~min}$ ), and mRNA was transcribed into fluorescent cRNA without 3 ' bias. Labeled cRNA were hybridized to the Human LncRNA Microarray, designed for 30,586 lncRNA and 26,109 mRNA. After washing with Gene Expression Wash Buffer 1 (Agilent Technologies, Inc.), and Gene Expression Wash Buffer 2 (Agilent Technologies, Inc.), the Agilent Scanner G2505C and Agilent feature extraction software (version 11.0.1.1; Agilent Technologies, Inc.) were used to scan and analyze the arrays. Quantile normalization and subsequent data processing were executed using the GeneSpring GX v12.0 software package (Agilent Technologies, Inc.). The microarray work was performed by Shanghai Kangchen Bio-tech, Inc., (Shanghai, China).

SYBR Green I RT-qPCR. The expression levels of differentially expressed lncRNA and mRNA were confirmed by RT-qPCR. Briefly, $5 \mu \mathrm{g}$ of total RNA from each sample was used for the synthesis of first strand cDNA using a PrimeScript ${ }^{\mathrm{TM}}$ RT Reagent Kit (Takara Biotechnology Co., Ltd., Dalian, China) according to the manufacturer's protocol. RT reaction was carried out in a $10 \mu \mathrm{l}$ reaction volume containing 5X PrimeScript ${ }^{\mathrm{TM}}$ Buffer, $1 \mu \mathrm{l} \mathrm{RT}$ specific primer, $0.5 \mu \mathrm{l}$ PrimeScript ${ }^{\mathrm{TM}}$ RT Enzyme Mix, $5 \mu \mathrm{g}$ of total RNA. RT assay was set at an initial denaturation step at $37^{\circ} \mathrm{C}$ for $15 \mathrm{~min}$, followed by $85^{\circ} \mathrm{C}$ for 5 sec. Following first strand cDNA synthesis, PCR reaction was performed in a $10 \mu 1$ reaction volume containing 1X SYBR Green PCR Master mix (Takara Biotechnology Co., Ltd.), $0.4 \mu \mathrm{M}$ of each specific forward and reverse primer, $0.5 \mu \mathrm{l}$ of cDNA template. PCR assay was set at an initial denaturation step at $95^{\circ} \mathrm{C}$ for $5 \mathrm{~min}$, followed by 40 cycles of $95^{\circ} \mathrm{C}$ for $15 \mathrm{sec}$ and $60^{\circ} \mathrm{C}$ for 1 min with an ABI 7500 Real-time PCR System (Applied Biosystems; Thermo Fisher Scientific, Inc.). The primers were as shown in Table II. The gene expression levels were normalized to that of GAPDH in cDNA samples, and all samples were measured in triplicate. The relative expression levels of the genes were calculated using the $\Delta \mathrm{Ct}$ method where $\Delta \mathrm{Ct}=\mathrm{Ct}$ median gene- $\mathrm{Ct}$ median GAPDH $(25,26)$.

Analysis of relationship between lncRNA and adjacent protein-coding genes. Target genes were predicted for 
Table I. Demographic, clinical and laboratory information of patients with RA.

\begin{tabular}{|c|c|c|c|c|c|c|c|}
\hline Subject & Sex & $\begin{array}{l}\text { Age, } \\
\text { years }\end{array}$ & DAS28 & $\begin{array}{l}\text { Erythrocyte sedimentation } \\
\text { rate, } \mathrm{mm} / \mathrm{h}\end{array}$ & $\begin{array}{l}\text { C-reactive } \\
\text { protein, } \mathrm{mg} / \mathrm{l}\end{array}$ & $\begin{array}{l}\text { Rheumatoid } \\
\text { factor, IU/ml }\end{array}$ & $\begin{array}{l}\text { Anti-cyclic citrullinated } \\
\text { peptide, } \mathrm{RU} / \mathrm{ml}\end{array}$ \\
\hline RA1 & Female & 55 & 5.03 & 37 & 8.30 & 142.00 & 670.70 \\
\hline RA2 & Female & 54 & 4.81 & 14 & 2.15 & 124.00 & 106.30 \\
\hline RA3 & Female & 43 & 5.50 & 12 & 2.04 & 46.50 & 155.60 \\
\hline RA4 & Male & 68 & 6.01 & 19 & 21.60 & $<20.00$ & 5.66 \\
\hline RA5 & Male & 37 & 6.39 & 33 & 16.10 & 59.60 & 20.61 \\
\hline RA6 & Female & 68 & 4.46 & 24 & 27.90 & $<20.00$ & 39.90 \\
\hline RA7 & Female & 68 & 4.12 & 10 & 5.02 & 115.00 & 175.10 \\
\hline RA8 & Female & 49 & 7.33 & 126 & 81.50 & $1,190.00$ & 493.60 \\
\hline RA9 & Female & 43 & 7.38 & 84 & 6.95 & 32.30 & 382.00 \\
\hline RA10 & Female & 52 & 5.25 & 40 & 4.06 & 80.90 & $1,600.00$ \\
\hline
\end{tabular}

RA, rheumatoid arthritis; DAS28, disease activity score 28.

Table II. LncRNA and mRNA gene primers for polymerase chain reaction.

\begin{tabular}{ll}
\hline Gene & \multicolumn{1}{c}{ Primer } \\
\hline ENST00000445339 & S: GATGGAACGAGGAATGGTAGCG A \\
& A: CAGTTCAGTGCATCGAGGTTTGC \\
ENST00000569920 & S: TAACATTCTGCATCCCTCACCCA \\
& A: CGCACATTACCTACCCCTTCTCA \\
ENST00000506982 & S: CAGTAAAAGAGCACTTGGTGGAA \\
& A: CCAGTGTTAGGGTCTGAGTCCAG \\
PGLYRP1 & S: CACATGAAGACACTGGGCTGGT \\
& A: CATGAAGCTGATGCCAATGGAC \\
LRRN3 & S: GGACAGCCTTTGTCAAGACTGAA \\
& A: CAGGGTGCAAACCTTTGGTG \\
CX3CR1 & S: AACTCCAGTAGCTTGGGACAAATCA \\
AAPDH & A: CCACAACTTGGGCACAGCA \\
& S: CCGGGAAACTGTGGCGTGATGG \\
& A: AGGTGGAGGAGTGGGTGTCGCTGTT
\end{tabular}

S, sense; A, antisense; lncRNA, long noncoding RNA.

differentially expressed lncRNAs. LncRNAs originate in complex transcriptional loci and regulate gene expression through epigenetic regulation of chromatin modification, and transcriptional and post-transcriptional processing (27). In order to determine the underlying roles of lncRNA in RA and shed light on the potential mechanisms of RA, the present study mainly focused on unveiling the relationship between IncRNA and mRNA. Abnormal lncRNA (fold-change, $\geq 3.0$ ) were subjected to bioinformatics analysis for target gene prediction. To increase the accuracy of target prediction, abnormal mRNA (fold-change, $\geq 3.0$ ) were integrated with the predicted IncRNA targets.

GO and KEGG pathway analysis based on differentially expressed mRNA. GO (www.geneontology.org) and KEGG (www.genome.ad.jp/kegg) databases were utilized to analyze biological functions and signaling pathways affected by abnormally expressed mRNA $(28,29)$. $\mathrm{P}<0.05$ was considered to indicate a statistically significant difference.

Statistical analysis. Statistical analysis and graphic presentation were performed using GraphPad Prism version 5.0 (GraphPad Software, Inc., La Jolla, CA, USA). A Student's t-test was used where the normality test passed; otherwise, the nonparametric Mann-Whitney $U$ test was used to analyze the data. $\mathrm{P}<0.05$ was considered to indicate a statistically significant difference.

\section{Results}

IncRNA and mRNA expression profiles in PBMCs of the different groups. To profile differentially expressed lncRNA in RA, a microarray analysis of IncRNA and mRNA expression in the RA group and healthy group was performed (Figs. 1 and 2). The results revealed that 139 lncRNA (88 up and 51 down) and 92 mRNA (67 up and 25 down) were significantly differentially expressed between the RA group and healthy group (fold-change $\geq 10.0 ; \mathrm{P}<0.05$ ). Additionally, 5,045 lncRNA (2,410 up and 2,635 down) and 3,289 mRNA (1,403 up and 1,886 down) were differentially expressed with a fold change $>2(\mathrm{P}<0.05)$. The most significantly deregulated lncRNA were ENST00000583574 (up fold-change, 257.7358) and ENST00000559539 (down fold-change, 4726.8003). The most significantly deregulated mRNA were ALAS2 (up fold-change, 52.16745) and KCNMB3 (down fold-change, 4999.96853) (data not shown).

$R T$-qPCR validation. To validate the reliability of the microarray results, three differentially expressed lncRNA and three differentially expressed mRNA were randomly selected and analyzed using RT-qPCR. For lncRNA, as demonstrated in Fig. 3, the results demonstrated that ENST00000445339 was significantly downregulated $(\mathrm{P}=0.042)$ andENST00000506982 was significantly upregulated $(\mathrm{P}=0.019)$ in the 24 patients with RA compared to the levels in the healthy group (Fig. 3A 
A

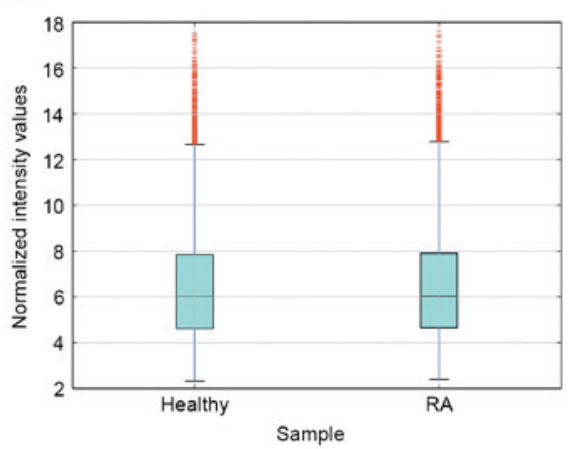

C

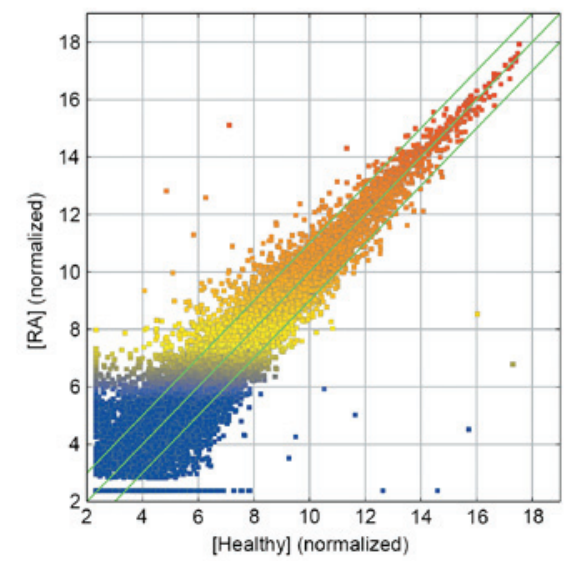

B

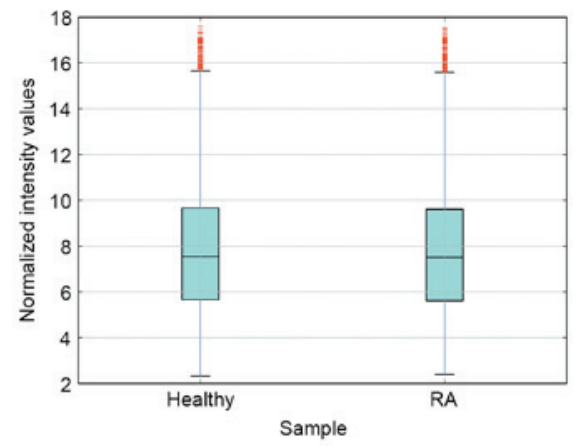

D

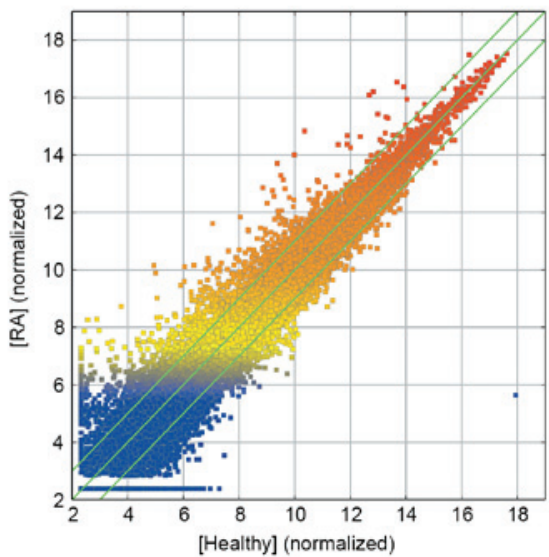

Figure 1. Box plots and scatter plots showing the variation in lncRNA and mRNA expression between the RA group and healthy group arrays. Box plots showing the distribution of the (A) lncRNA and (B) mRNA profiles in the RA and healthy groups. Following normalization, the distributions of the log2-ratios among the tested samples were nearly the same. The scatter plots of (C) lncRNA and (D) mRNA were used for visualization to assess the variation between the RA group and healthy group arrays. The $\mathrm{x}$ - and y-axes in the scatter plots represent the normalized signal values of each group (log2 scaled). The lncRNA and mRNA above the top green line and below the bottom green line are those with a 2-fold-change in expression between groups. lncRNA, long non-coding RNA; RA, rheumatoid arthritis.

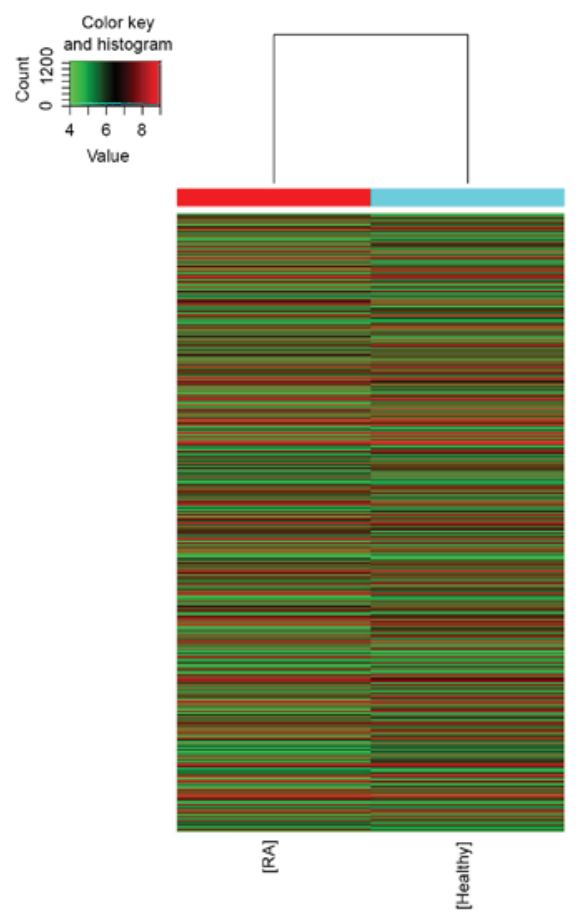

Figure 2. Hierarchical clustering analysis of lncRNA and mRNA profile comparison between the RA group $(\mathrm{n}=10)$ and healthy group $(\mathrm{n}=10)$. $\operatorname{lncRNA}$ long non-coding RNA; RA, rheumatoid arthritis. and C, respectively). No significant difference was observed in the expression of ENST00000569920 between RA patients and healthy controls $(\mathrm{P}=0.57$; Fig. 3B). For mRNA, the expression of PGLYRP1 was significantly downregulated $(\mathrm{P}<0.0001), \mathrm{LRRN} 3$ was significantly upregulated $(\mathrm{P}=0.0003)$ and CX3CR1 was significantly upregulated $(\mathrm{P}=0.048)$ in patients with RA compared with the levels in healthy controls (Fig. 3D-F). This demonstrated that RT-qPCR results were consistent with those of the microarray analysis.

IncRNA classification and subgroup analysis. Evidence from previous reports has indicated that lncRNA may be classified into lncRNA with enhancer-like functions, antisense lncRNA and lincRNA $(26,30)$. The results of the present study indicated that 610 intergenic lncRNA (304 upregulated and 306 downregulated), 251 enhancer-like lncRNA (138 upregulated and 113 downregulated) and 134 antisense lncRNA (57 upregulated and 77 downregulated) were differentially expressed (fold-change $\geq 2.0 ; \mathrm{P}<0.05$ ) between the RA group and healthy group. Some nearby coding genes that may be regulated by these differentially expressed lncRNA were also identified (data not shown).

Integration of differentially expressed mRNA into the predicted targets of differentially expressed lncRNA. The 
A

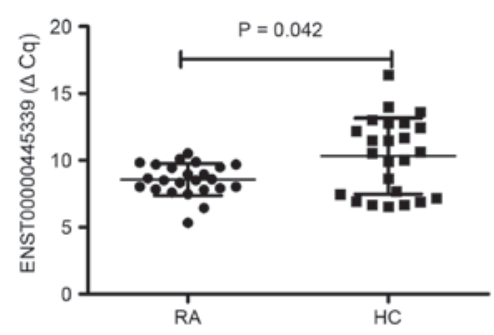

C

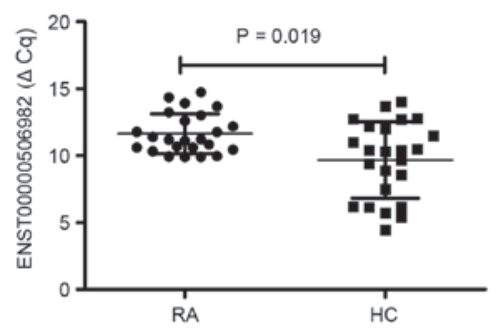

$\mathrm{E}$

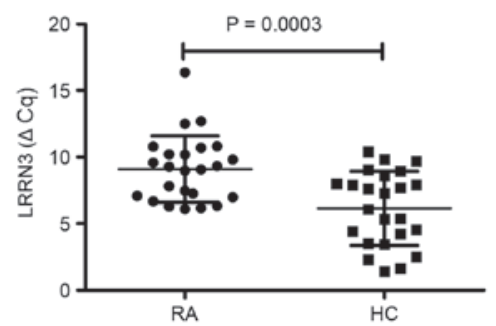

B

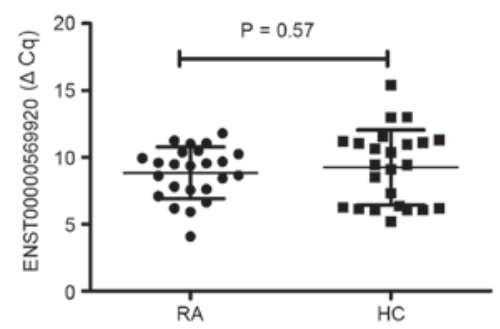

D

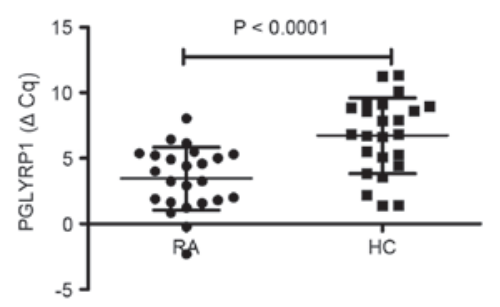

$\mathrm{F}$

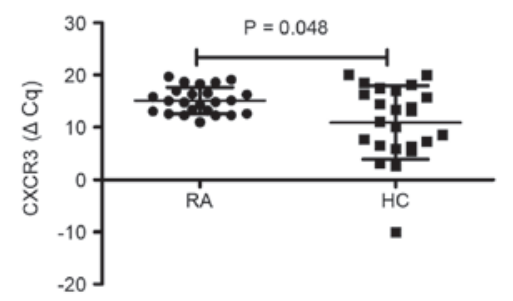

Figure 3. Relative expression levels of lncRNA and mRNA in 24 paired RA patients and HC individuals determined by reverse transcription-quantitative polymerase chain reaction. (A) ENST00000445339 was demonstrated to be significantly downregulated in RA patients compared with the level in the HC group. (B) No significant difference was observed in the expression of ENST00000569920 between RA patients and healthy controls. (C) ENST00000506982 was identified as significantly upregulated in RA patients compared with the level in HCs. (D) PGLYRP1 was demonstrated to be significantly downregulated in RA patients compared with the level in the HC group. (E) LRRN3 was identified as significantly upregulated in RA patients compared with the level in the HC group. (F) CX3CR1 was identified as significantly upregulated in RA patients compared with the level in the HC group. The expression level of lncRNA and mRNA were normalized using GAPDH as an internal control. lncRNA, long non-coding RNA; RA, rheumatoid arthritis; HC, healthy control.

present results revealed that the majority of differentially expressed lncRNA were from intergenic regions (42\%), natural antisense to protein-coding loci (19\%), or intronic antisense to protein-coding loci (15\%), with the others representing intronic sense-overlapping, exon sense-overlapping, and bidirectional regions. In addition, 2,859 of 5,045 differentially expressed lncRNA were oriented in or around a known protein-coding region (i.e. not intergenic). Therefore, differentially expressed lncRNA (fold-change, $\geq 3.0$ ) were integrated with predicted mRNA (fold-change, $\geq 3.0$ ). The result demonstrated that 109 differentially expressed protein-coding genes were markedly associated with each of the 85 differentially expressed lncRNA to generate 135 lncRNA-mRNA target pairs. Among them, 81 pairs were differentially expressed unidirectionally (upregulated or downregulated), while 54 pairs were differentially expressed bidirectionally (data not shown).

GO and KEGG pathway analysis of deregulated $m R N A$. Through GO analysis, it was demonstrated that these abnormal transcripts of lncRNA were associated with biological process, cellular components and molecular function (Fig. 4). The analysis indicated that the differentially expressed mRNA between the RA group and healthy group were significantly enriched $(\mathrm{P}<0.05)$ in response to wounding, DNA packaging complex, chemokine activity, RNA metabolic process, nuclear lumen and DNA binding. Pathway analysis indicated that 23 upregulated pathways were identified, including systemic lupus erythematosus and alcoholism pathways. A total of 23 downregulated pathways were identified, including bile secretion and $\mathrm{T}$ cell receptor signaling pathway (Fig. 5).

\section{Discussion}

Over the past decades, expression profiles of genes involved in the pathogenesis of RA have been extensively explored $(31,32)$. However, the molecular regulatory mechanisms underlying RA have not been fully elucidated. Increasing evidence indicates that lncRNA may serve crucial roles in various biological processes and may regulate gene expression in human disease $(33,34)$. Few studies have reported that lncRNA have an important role in RA (16-20); however, fewer studies have investigated the expression profile of lncRNA in RA (16-20). Therefore, the present study aimed to better determine the potential role of IncRNA in contributing to RA.

PBMCs are crucial sentinels of host defense response, being used to identify novel disease mediators, disease variants 
A

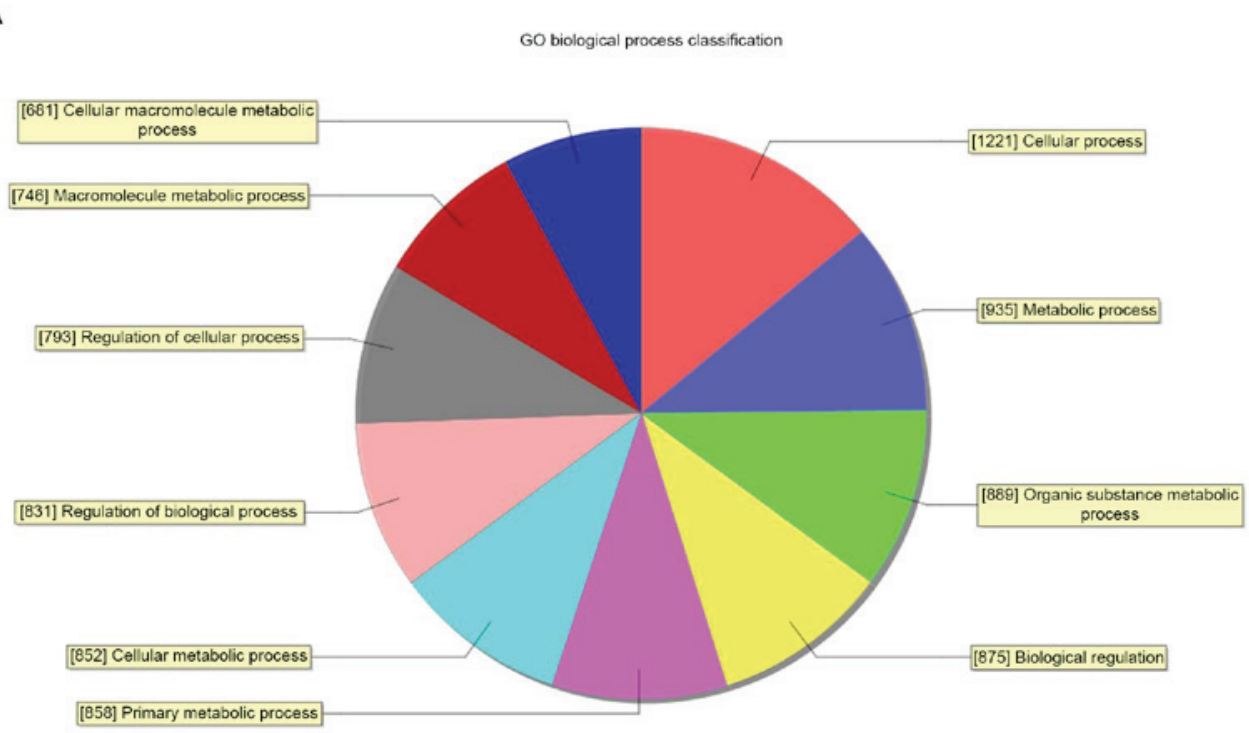

B

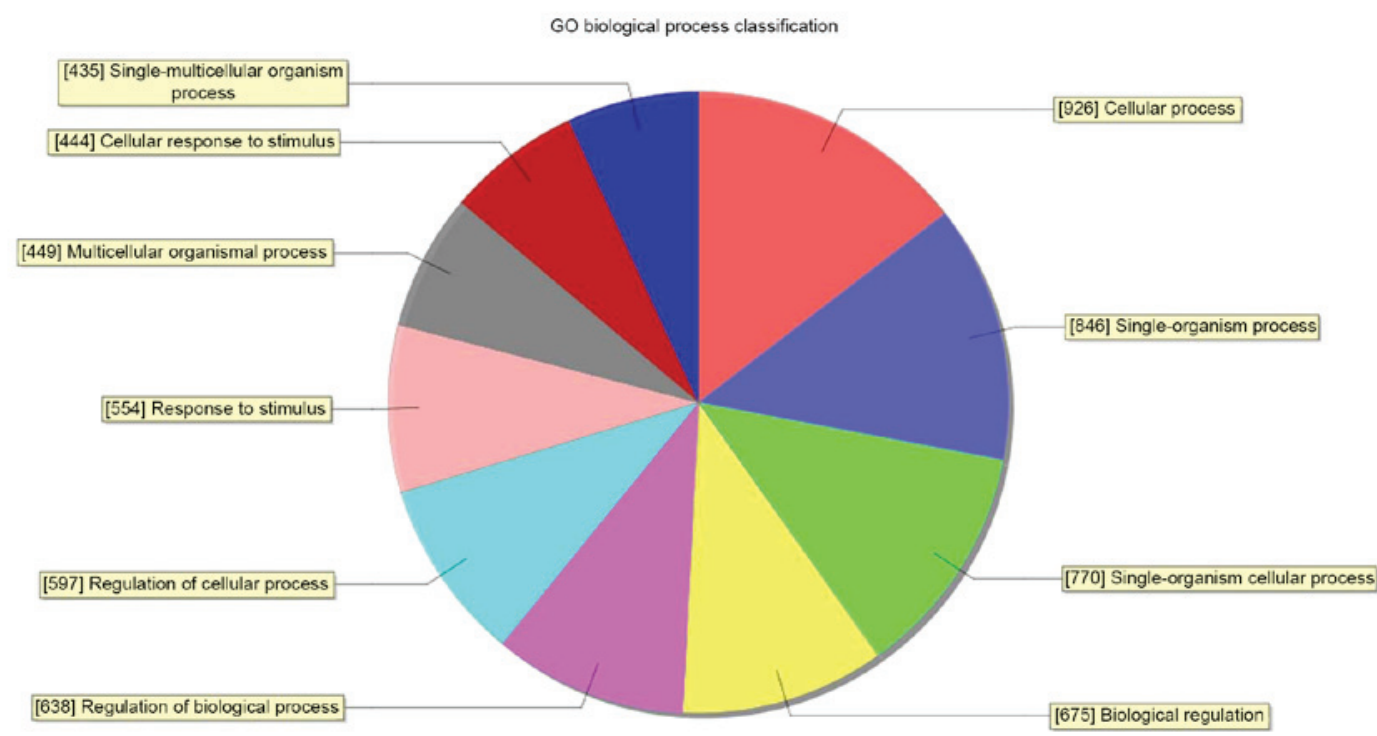

C

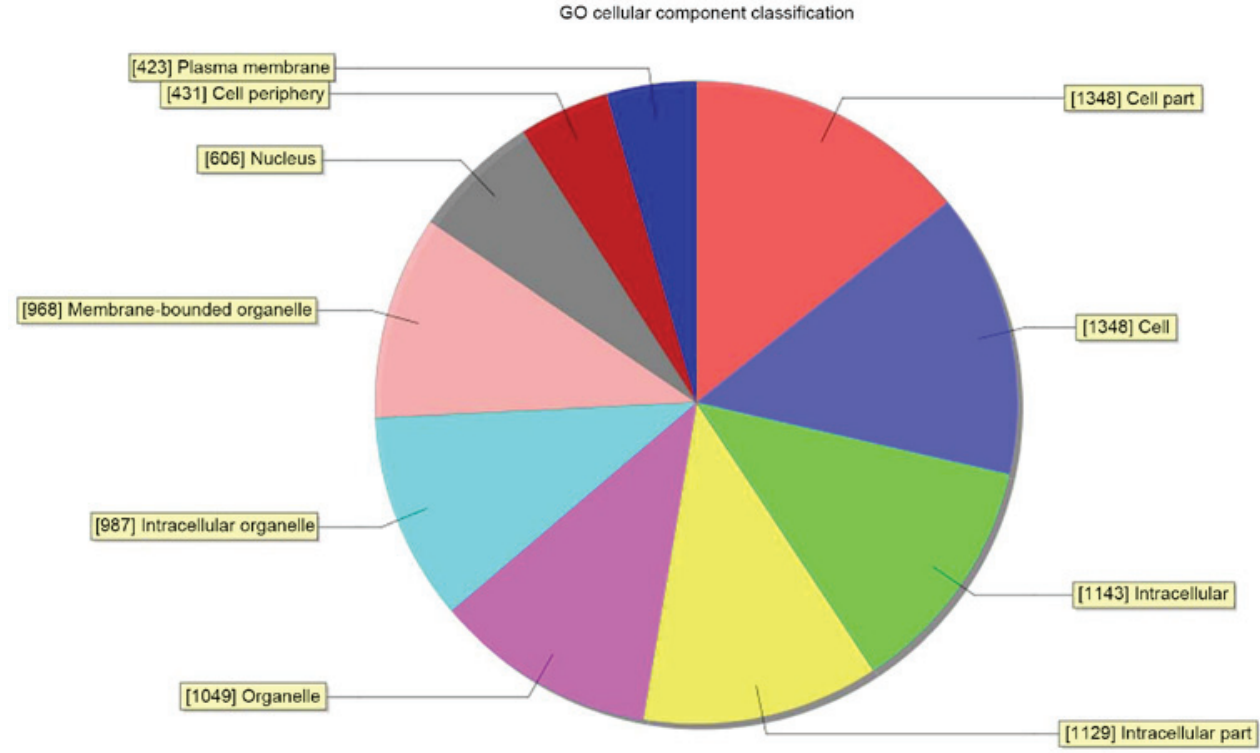

Figure 4. GO enrichment analysis for differentially regulated mRNA. GO analysis of (A) downregulated and (B) upregulated mRNA according to biological process. $\mathrm{GO}$ analysis of $(\mathrm{C})$ downregulated. 
D

GO cellular component classification

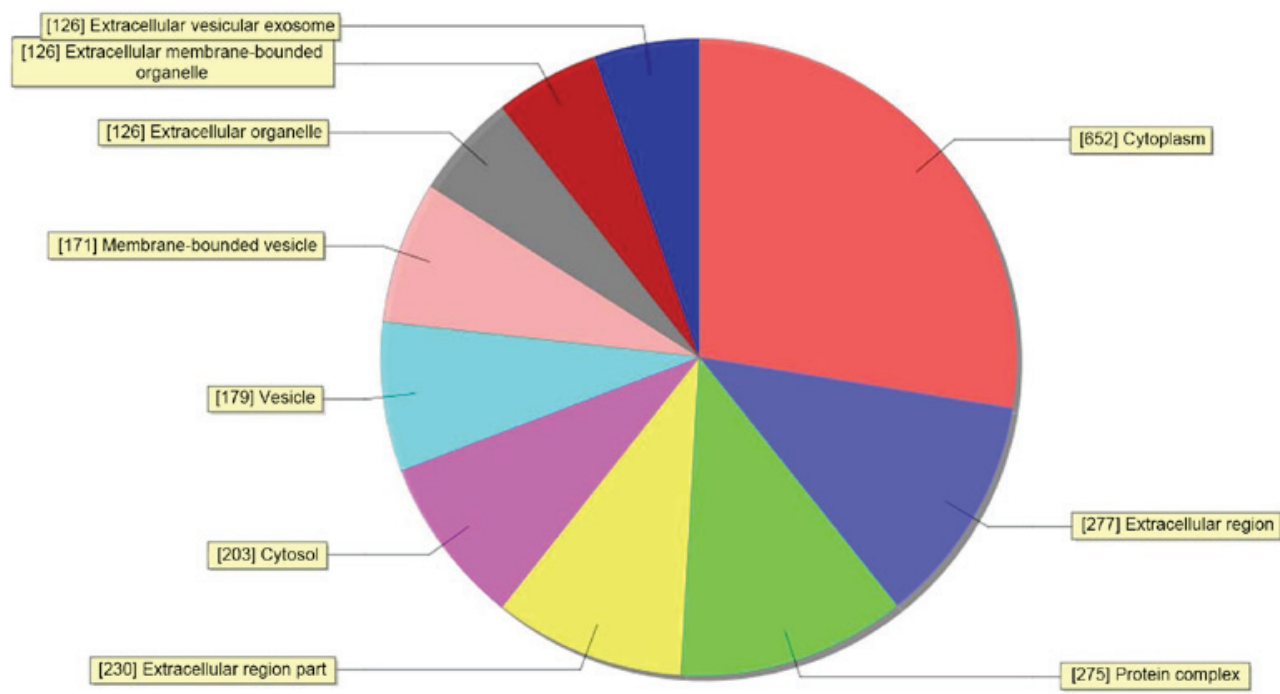

E

GO molecular function classification

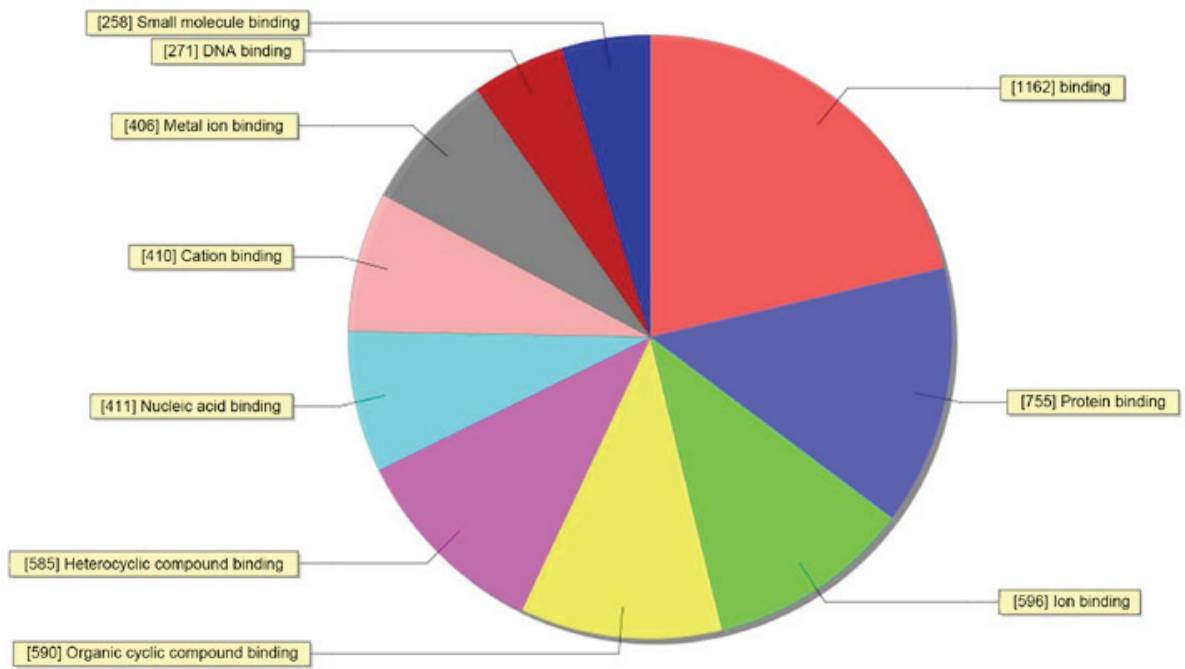

$\mathrm{F}$

GO molecular function classification

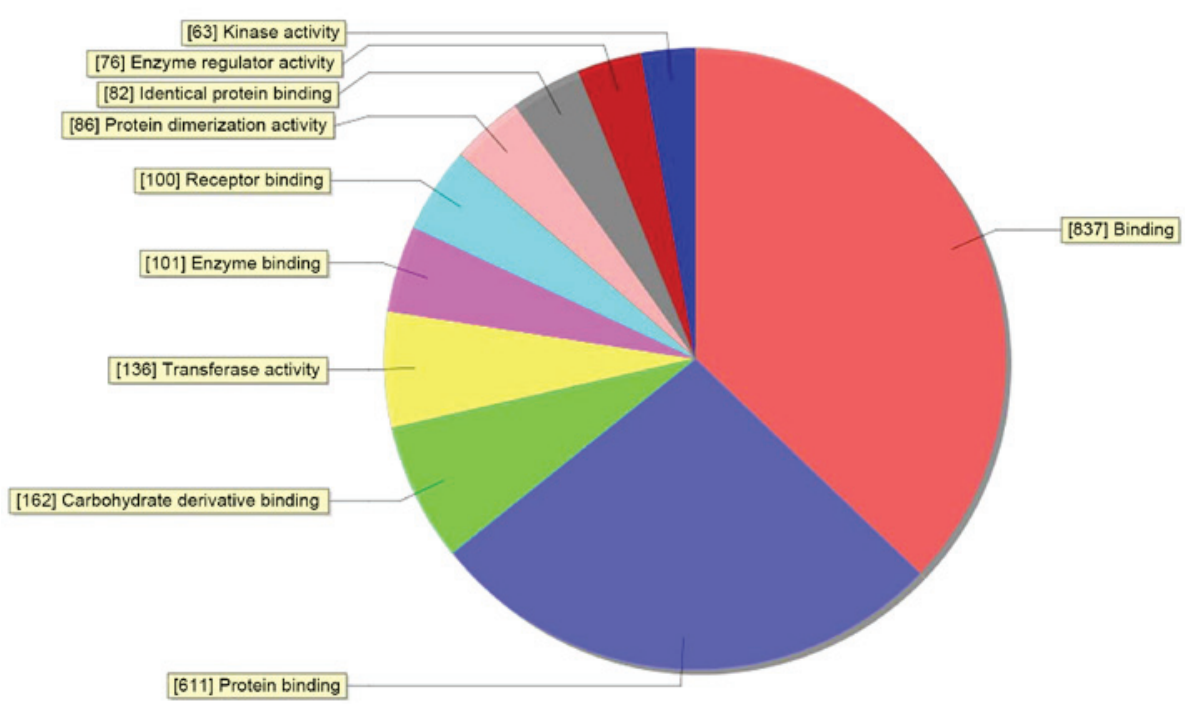

Figure 4. Continued. (D) Upregulated mRNA according to cellular component. GO analysis of (E) downregulated and (F) upregulated mRNA according to molecular function. GO, Gene Ontology. 
A

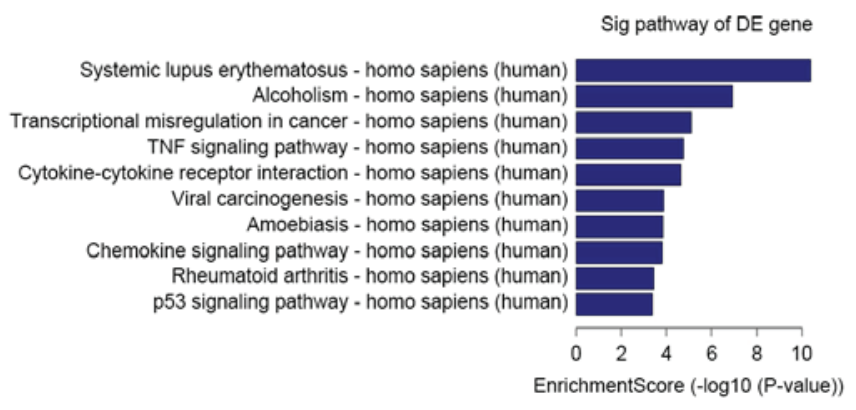

B

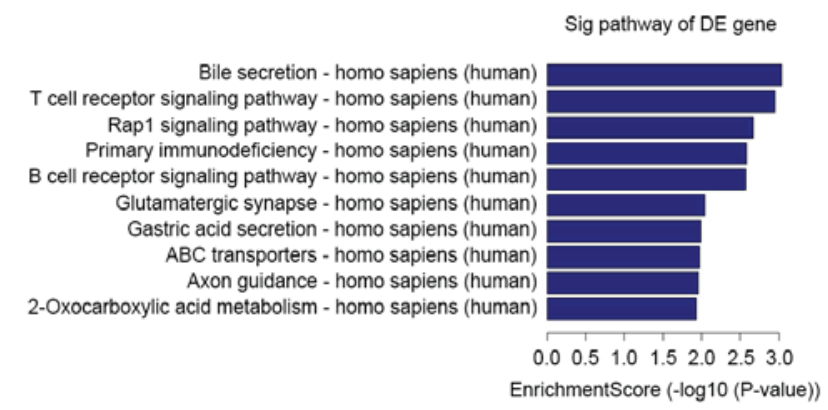

Figure 5. Pathway analysis of upregulated and downregulated mRNA in rheumatoid arthritis. (A) A total of 23 upregulated pathways were identified, including systemic lupus erythematosus and alcoholism. (B) A total of 23 downregulated pathways were identified, including bile secretion and T cell receptor signaling pathway. DE, differently expressed; sig, significant.

and treatment responses (35). Microarray technology has been used to distinguish differences in gene expression profiles in PBMCs of RA $(8,32)$. In addition, PBMCs may simulate the conditions of synovial tissue in RA, thus making it possible to bypass the requirement for synovial tissue specimen, allowing the research of larger patient populations (9). The present study analyzed the gene expression patterns of PBMCs in patients with RA using microarrays to uncover the possible role of lncRNA in the pathogenesis of this disease. The results demonstrated that, compared with the healthy group, there were notable changes in the expression of 1ncRNA (2,410 upregulated and 2,635 downregulated) and mRNA (1,403 upregulated and 1,886 downregulated) in PBMCs. Furthermore, 135 matched IncRNA-mRNA pairs were identified for 85 differentially expressed lncRNA and 109 differentially expressed mRNA. The present study then focused on six altered lncRNA and mRNA and assessed their expression level and the microarray results via RT-qPCR. To the best of our knowledge, only one report exists regarding the lncRNA pattern of RA using IncRNA array (17). The previous study analyzed the expression profiles of 83 lncRNA and revealed that 18 lncRNA were abnormal (17). However, 83 lncRNA are only a fraction of the lncRNA family, and other lncRNA have yet to be identified. Additionally, the present study comprehensively analyzed the expression profiles of lncRNA and mRNA, using Arraystar Human LncRNA Microarray V3.0, for 30,586 lncRNA and 26,109 mRNA.

As we know, there are five kinds of lncRNA, including sense, antisense, bidirectional, intronic and intergenic. Various studies have revealed a variety of mechanisms by which IncRNA function; however, a common and key function of lncRNA is to alter the expression of nearby encoding genes by impacting the process of transcription (36) or directly serving a role as enhancer-like elements $(30,37)$. The present study identified notably different expression patterns of enhancer-like lncRNA (138 upregulated and 113 downregulated), intergenic lncRNA (304 upregulated and 306 downregulated) and antisense lncRNA (57 upregulated and 77 downregulated). In addition, the present study identified some nearby coding genes that may be regulated by lincRNA and enhancer-like lncRNA, and some coding transcripts may be regulated by antisense lncRNA. These results indicated that lncRNA could function as lincRNA, enhancer-like lncRNA and antisense lncRNA in RA.
Furthermore, the present study revealed that not all lncRNAs that were differentially expressed also exhibited differential expression in their associated protein-coding genes. This result suggested that these abnormal RA-associated lncRNA and mRNA may be independently regulated. Future studies are required to uncover their potential functional roles in RA disease by RNA interference or overexpression methods in an appropriate model system. However, the majority of the differentially expressed lncRNA associated protein-coding genes were not deregulated, suggesting that more complex mechanisms of regulation existed for these lncRNA.

To understand the underlying roles of lncRNA, GO category and KEGG pathway analyses were utilized to determine the target gene pool. These results predicted that downregulated and upregulated transcripts of IncRNA were associated with biological process, cellular component and molecular function, which were associated with 46 gene pathways that corresponded to transcripts, including 'rheumatoid arthritis' and 'TNF signaling pathway'. These pathways may have essential roles in the occurrence and development of RA.

The present study only detected one RA group and one control group by microassay of gene chip. This is indeed a limitation of the study. In order to minimize the variation between groups, the chip assay was performed in the mode of a pooling test, according to previous reports $(26,38)$ in the same research field. In future studies, we intend to investigate the microassay of gene chip in a larger sample size in triplicate.

In conclusion, the present study described the global expression profiling of IncRNA and mRNA in RA by microarray technology for the first time, which opened up a new and interesting pathway to provide novel insights into the relationship between lncRNA in PBMCs and RA. Additionally, the roles for the abnormal lncRNA in the regulation of the TNF signaling pathway and RA signaling pathways were researched.

\section{Acknowledgements}

The present study was financially supported by the National Natural Science Foundation of China (grant no. 81360459) and Jiangxi Provincial Natural Science Foundation of China (grant no. 20151BAB215031). We are grateful to Dr Rui Wu (Department of Rheumatology, The First Affiliated Hospital of Nanchang University, Nanchang, China) for providing help. 


\section{References}

1. Smolen JS, Aletaha D, Koeller M, Weisman MH and Emery P: New therapies for treatment of rheumatoid arthritis. Lancet 370 : 1861-1874, 2007

2. McInnes IB and Schett G: The pathogenesis of rheumatoid arthritis. N Engl J Med 365: 2205-2219, 2011.

3. Djebali S, Davis CA, Merkel A, Dobin A, Lassmann T, Mortazavi A, Tanzer A, Lagarde J, Lin W, Schlesinger F, et al: Landscape of transcription in human cells. Nature 489: 101-108, 2012.

4. Mehler MF and Mattick JS: Noncoding RNAs and RNA editing in brain development, functional diversification, and neurological disease. Physiol Rev 87: 799-823, 2007.

5. KapranovP,Cheng J,DikeS,Nix DA,Duttagupta R,WillinghamAT, Stadler PF, Hertel J, Hackermüller J, Hofacker IL, et al: RNA maps reveal new RNA classes and a possible function for pervasive transcription. Science 316: 1484-1488, 2007.

6. Novikova IV, Hennelly SP and Sanbonmatsu KY: Sizing up long non-coding RNAs: Do IncRNAs have secondary and tertiary structure? Bioarchitecture 2: 189-199, 2012

7. Kowalczyk MS, Higgs DR and Gingeras TR: Molecular biology: RNA discrimination. Nature 482: 310-311, 2012

8. Long L, Yu P, Liu Y, Wang S, Li R, Shi J, Zhang X, Li Y, Sun X, Zhou B, et al: Upregulated microRNA-155 expression in peripheral blood mononuclear cells and fibroblast-like synoviocytes in rheumatoid arthritis. Clin Dev Immunol 2013: 296139, 2013.

9. Pauley KM, Satoh M, Chan AL, Bubb MR, Reeves WH and Chan EK: Upregulated miR-146a expression in peripheral blood mononuclear cells from rheumatoid arthritis patients. Arthritis Res Ther 10: R101, 2008.

10. Dong L, Wang X, Tan J, Li H, Qian W, Chen J, Chen Q, Wang J, $\mathrm{Xu}$ W, Tao C and Wang S: Decreased expression of microRNA-21 correlates with the imbalance of Th17 and Treg cells in patients with rheumatoid arthritis. J Cell Mol Med 18: 2213-2224, 2014.

11. Ponting CP, Oliver PL and Reik W: Evolution and functions of long noncoding RNAs. Cell 136: 629-641, 2009.

12. Wang KC and Chang HY: Molecular mechanisms of long noncoding RNAs. Mol Cell 43: 904-914, 2011.

13. Zhu J, Liu S, Ye F, Shen Y, Tie Y, Zhu J, Jin Y, Zheng X, Wu Y and $\mathrm{Fu} \mathrm{H}$ : The long noncoding RNA expression profile of hepatocellular carcinoma identified by microarray analysis. PLoS One 9: e101707, 2014.

14. Wan ZY, Song F, Sun Z, Chen YF, Zhang WL, Samartzis D, Ma CJ, Che L, Liu X, Ali MA, et al: Aberrantly expressed long noncoding RNAs in human intervertebral disc degeneration: A microarray related study. Arthritis Res Ther 16: 465, 2014.

15. Yang B, Xia ZA, Zhong B, Xiong X, Sheng C, Wang Y, Gong W, Cao Y, Wang Z and Peng W: Distinct hippocampal expression profiles of long non-coding RNAs in an alzheimer's disease model. Mol Neurobiol 54: 4833-4846, 2017.

16. Stuhlmüller B, Kunisch E, Franz J, Martinez-Gamboa L, Hernandez MM, Pruss A, Ulbrich N, Erdmann VA, Burmester GR and Kinne RW: Detection of oncofetal h19 RNA in rheumatoid arthritis synovial tissue. Am J Pathol 163: 901-911, 2003.

17. Song J, Kim D, Han J, Kim Y, Lee M and Jin EJ: PBMC and exosome-derived Hotair is a critical regulator and potent marker for rheumatoid arthritis. Clin Exp Med 15: 121-126, 2015.

18. Spurlock CF III, Tossberg JT, Matlock BK, Olsen NJ and Aune TM: Methotrexate inhibits NF- $\mathrm{KB}$ activity via long intergenic (noncoding) RNA-p21 induction. Arthritis Rheumatol 66: 2947-2957, 2014

19. Messemaker TC, Frank-Bertoncelj M, Marques RB, Adriaans A, Bakker AM, Daha N, Gay S, Huizinga TW, Toes RE, Mikkers HM and Kurreeman F: A novel long non-coding RNA in the rheumatoid arthritis risk locus TRAF1-C5 influences C5 mRNA levels. Genes Immun 17: 85-92, 2016.

20. Lu MC, Yu HC, Yu CL, Huang HB, Koo M, Tung CH and Lai NS Increased expression of long noncoding RNAs LOC100652951 and LOC100506036 in T cells from patients with rheumatoid arthritis facilitates the inflammatory responses. Immunol Res 64 576-583, 2016.

21. Arnett FC, Edworthy SM, Bloch DA, McShane DJ, Fries JF, Cooper NS, Healey LA, Kaplan SR, Liang MH and Luthra HS: The American Rheumatism Association 1987 revised criteria for the classification of rheumatoid arthritis. Arthritis Rheum 31: 315-324, 1988
22. Prevoo ML, van't Hof MA, Kuper HH, van Leeuwen MA, van de Putte LB and van Riel PL: Modified disease activity scores that include twenty-eight-joint counts. Development and validation in a prospective longitudinal study of patients with rheumatoid arthritis. Arthritis Rheum 38: 44-48, 1995.

23. Hashemi R, Majidi A, Motamed H, Amini A, Najari F and Tabatabaey A: Erythrocyte sedimentation rate measurement using as a rapid alternative to the westergren method. Emerg (Tehran) 3: 50-53, 2015.

24. Zhang J, Cui X, Shen Y, Pang L, Zhang A, Fu Z, Chen J, Guo X, Gan W and Ji C: Distinct expression profiles of LncRNAs between brown adipose tissue and skeletal muscle. Biochem Biophys Res Commun 443: 1028-1034, 2014.

25. Tian M, Chen R, Li T and Xiao B: Reduced expression of circRNA hsa circ 0003159 in gastric cancer and its clinical significance. J Clin Lab Anal: Jun 15, 2017 (Epub ahead of print). doi: $10.1002 /$ jcla.22281.

26. Xu G, Chen J, Pan Q, Huang K, Pan J, Zhang W, Chen J, Yu F, Zhou $\mathrm{T}$ and Wang Y: Long noncoding RNA expression profiles of lung adenocarcinoma ascertained by microarray analysis. PLoS One 9: e104044, 2014.

27. Tsai MC, Manor O, Wan Y, Mosammaparast N, Wang JK, Lan F, Shi Y, Segal E and Chang HY: Long noncoding RNA as modular scaffold of histone modification complexes. Science 329: 689-693, 2010.

28. Kanehisa M, Goto S, Furumichi M, Tanabe M and Hirakawa M: KEGG for representation and analysis of molecular networks involving diseases and drugs. Nucleic Acids Res 38: D355-D360, 2010.

29. Zhang T, Jiang M, Chen L, Niu B and Cai Y: Prediction of gene phenotypes based on GO and KEGG pathway enrichment scores. Biomed Res Int 2013: 870795, 2013.

30. Ørom UA, Derrien T, Beringer M, Gumireddy K, Gardini A, Bussotti G, Lai F, Zytnicki M, Notredame C, Huang Q, et al: Long noncoding RNAs with enhancer-like function in human cells. Cell 143: 46-58, 2010

31. Teixeira VH, Olaso R, Martin-Magniette ML, Lasbleiz S, Jacq L, Oliveira CR, Hilliquin P, Gut I, Cornelis F and Petit-Teixeira E: Transcriptome analysis describing new immunity and defense genes in peripheral blood mononuclear cells of rheumatoid arthritis patients. PLoS One 4: e6803, 2009.

32. van der Pouw Kraan TC, Wijbrandts CA, van Baarsen LG, Voskuyl AE, Rustenburg F, Baggen JM, Ibrahim SM, Fero M, Dijkmans BA, Tak PP and Verweij CL: Rheumatoid arthritis subtypes identified by genomic profiling of peripheral blood cells: Assignment of a type I interferon signature in a subpopulation of patients. Ann Rheum Dis 66: 1008-1014, 2007.

33. Mercer TR, Dinger ME and Mattick JS: Long non-coding RNAs: Insights into functions. Nat Rev Genet 10: 155-159, 2009.

34. Gupta RA, Shah N, Wang KC, Kim J, Horlings HM, Wong DJ, Tsai MC, Hung T, Argani P, Rinn JL, et al: Long noncoding RNA HOTAIR reprograms chromatin state to promote cancer metastasis. Nature 464: 1071-1076, 2010.

35. Toonen EJ, Barrera P, Radstake TR, van Riel PL, Scheffer H, Franke B and Coenen MJ: Gene expression profiling in rheumatoid arthritis: Current concepts and future directions. Ann Rheum Dis 67: 1663-1669, 2008.

36. Mattick JS and Gagen MJ: The evolution of controlled multitasked gene networks: The role of introns and other noncoding RNAs in the development of complex organisms. Mol Biol Evol 18: 1611-1630, 2001.

37. Mattick JS: Linc-ing Long noncoding RNAs and enhancer function. Dev Cell 19: 485-486, 2010

38. Yi Z, Li J, Gao K and Fu Y: Identifcation of differentially expressed long non-coding RNAs in CD4+ T cells response to latent tuberculosis infection. J Infect 69: 558-568, 2014. 